STUDI

FRANCESI

\section{Studi Francesi}

Rivista quadrimestrale fondata da Franco Simone

170 (LVII | II) | 2013

Varia

\title{
Lectures de La Fontaine. Le recueil de 1668, sous la direction de Christine Noille
}

\section{Federico Corradi}

\section{(2) OpenEdition}

1 Journals

\section{Edizione digitale}

URL: https://journals.openedition.org/studifrancesi/3065

DOI: $10.4000 /$ studifrancesi.3065

ISSN: 2421-5856

\section{Editore}

Rosenberg \& Sellier

\section{Edizione cartacea}

Data di pubblicazione: 1 juillet 2013

Paginazione: 448-449

ISSN: 0039-2944

\section{Notizia bibliografica digitale}

Federico Corradi, «Lectures de La Fontaine. Le recueil de 1668, sous la direction de Christine Noille»,

Studi Francesi [Online], 170 (LVII | II) | 2013, online dal 30 novembre 2015, consultato il 31 janvier 2023. URL: http://journals.openedition.org/studifrancesi/3065; DOI: https://doi.org/10.4000/studifrancesi. 3065

Questo documento è stato generato automaticamente il 31 janvier 2023.

\section{(c)}

Creative Commons - Attribuzione - Non commerciale - Non opere derivate 4.0 Internazionale - CC BYNC-ND 4.0

https://creativecommons.org/licenses/by-nc-nd/4.0/ 


\section{Lectures de La Fontaine. Le recueil de 1668 , sous la direction de Christine Noille}

Federico Corradi

\section{NOTIZIA}

Lectures de La Fontaine. Le recueil de 1668, sous la direction de christine NOILLE, Presses Universitaires de Rennes, 2011, Collection «Didact Français», pp. 331.

1 Il presente volume si presenta come un doppio percorso: nell'universo testuale delle Fables e nel paesaggio critico contemporaneo a cui il capolavoro di La Fontaine ha dato origine. L'occasione è l'inserimento delle Fables nel programma dell'agrégation 2012, che sancisce la perdurante fortuna accademica e scolastica di un classico percepito come sottilmente e durevolmente contemporaneo. La capacità che quest'opera dimostra di caricarsi di sempre nuovi sottintesi con il variare delle mode critiche e delle opzioni filosofiche non era certo più da dimostrare, ma la varietà dei contributi riuniti in questo libro, opera di studiosi giovani e meno giovani, ne fornisce un'ulteriore prova. Gli articoli spaziano dalla metrica, un aspetto decisivo eppure stranamente trascurato dagli studi recenti, ai dispositivi retorici attivati negli apologhi, dalle categorie politiche e filosofiche agli aspetti filologici e agli studi tematici. Il piano filologico non è da trascurare: la curatrice del volume evidenzia fin dall'avant-propos la contraddizione insita nella scelta di mettere in programma "la raccolta del 1668" rinviando però ad un'edizione, quella curata da Jean-Charles Darmon e Sabine Gruffat per «Le Livre de Poche», che riproduce il testo del 1692 , testo che comporta diverse modifiche rispetto all'edizione originale, a cominciare dall'assenza delle illustrazioni. L'articolo di Alain CANTILLON approfondisce la discussione polemica intorno a questa scelta. Prescindere da una valutazione accurata delle differenti edizioni di un classico significa secondo lui porlo in una sorta di presente atemporale, farne l'espressione di una pretesa saggezza senza tempo secondo un processo di idealizzazione acritica che non è privo di sottintesi 
politici, come dimostra l'interpretazione che dell'opera di La Fontaine ha dato Marc Fumaroli. L'analisi delle differenze materiali tra le raccolte del 1668 e del 1692 mostra che si tratta di due opere diverse e non di una prima manifestazione parziale e poi di una completa di un'unica entità testuale. Cantillon polemizza inoltre con le edizioni moderne che, col pretesto di rendere accessibile un testo alle abitudini moderne di lettura, di fatto lo riscrivono. L'articolo di Cantillon presenta quindi con grande efficacia alcune delle conseguenze aberranti della canonizzazione di un classico.

Della metrica delle Fables si occupano i due saggi di Guillaume PEUREUX e di Benoît DE CORNULIER. Molto interessanti le osservazioni di Peureux, che mostra come la "mise en vers" sia tutt'altro che un'operazione accessoria per La Fontaine. Il suo uso del verso mostra una presa di distanza rispetto all'estrema prevedibilità della metrica classica. Si può esitare, nell'analizzare la metrica delle Fables, tra l'identificazione di schemi strofici classici, che comporterebbero però una sfasatura tra metrica e sintassi, o di moduli più antichi, preclassici o popolari, che rispettano maggiormente le partizioni della sintassi. Questo gioco tra metrica e sintassi favorisce la fluidità narrativa a detrimento della riconoscibilità immediata degli schemi. Anche le numerose négligences spesso rimproverate a La Fontaine producono un effetto di straniamento che avvicina la poesia alla prosa e compensa la noia potenziale del lettore di fronte ad un corpus di storie usurate dal tempo. Anche Benoît de Cornulier evidenzia da una parte la libertà metrica che La Fontaine persegue, dall'altra il ricorrere di alcune regole di base, come la regola "des deux couleurs" e l'alternanza di rime maschili e femminili. L'assenza, insomma, di schemi regolari e prevedibili non impedisce che ogni favola presenti una continuità metrica, anche se diverse interpretazioni sono sempre possibili nell'identificazione delle unità. Uno degli effetti che La Fontaine ottiene è di far vacillare i limiti tra un'unità metrica e l'altra con un effetto di legato propizio alla fluidità narrativa.

3 Altri saggi del volume indagano il piano retorico ed ermeneutico. Marc EsCoLA analizza la riscrittura in versi di alcuni apologhi già raccontati in prosa nella vita di Esopo. La riscrittura in versi sottrae la favola al suo contesto di enunciazione e quindi opacizza il suo significato politico, che però è sempre possibile per il lettore riattivare autonomamente. Molto più interessante l'articolo di Alain BRUNN, che si interroga sull'istanza autoriale nelle Fables, proponendo un confronto con gli altri moralisti. Se La Rochefoucauld nelle Maximes enuncia autorevolmente le sue verità senza che appaia esplicitamente una voce di autore, nelle Pensées sono i signori di Port-Royal, editori dell'opera, che fanno di Pascal un'autorità scientifica e filosofica. La Bruyère si nasconde invece dietro l'autorità di Teofrasto. La Fontaine, che non è né un aristocratico di alto rango, né un erudito, sceglie la via della discrezione. L'io d'autore nella prima raccolta compare soprattutto nell'articolazione tra racconto e morale, talvolta per mostrare la sfasatura tra i due piani. Delega spesso la responsabilità del suo discorso ad altre voci (è quella che Brunn chiama dénégation d'autorité), assumendo la posizione di distacco di un interprete che sperimenta diverse interpretazioni piuttosto che fornirne un'unica valida. Si fa così maestro di lettura prima e più che maestro di costumi. Di grande interesse anche il saggio della curatrice del volume, Christine NoILLE, che affronta un problema chiave, quello della struttura della raccolta. Se è certo che ogni favola rappresenta un'unità di senso autosufficiente, è anche chiaro che il suo inserimento in un insieme testuale più ampio non può non interferire nell'interpretazione. Noille elenca diversi modelli di organizzazione a cui La Fontaine 
poteva ricorrere: il modello della lista, proprio delle raccolte esopiche, il modello del libro, autorizzato da Fedro, il modello della raccolta di poesie e il modello del "beau désordre", adottato da La Rochefoucauld nelle Maximes. Le Fables alludono per certi aspetti a ognuno di questi modelli, ma nessuno di essi è sufficiente da solo a spiegarne l'ordine. Come mostra l'analisi della struttura interna di ogni libro, gli effetti ricercati da La Fontaine nell'accostamento o nel richiamo a distanza tra gli apologhi non sono mai sistematizzati e richiedono sempre uno sforzo interpretativo da parte del lettore.

La ditione politica delle Fables è invece interrogata da Arnaud WELFRINGER. Partendo dalla constatazione che quest'opera ha autorizzato negli anni le letture politiche più divergenti, l'A. propone di spostare la discussione su un altro piano: partendo da una reinterpretazione di «Simonide préservé par les dieux», Welfringer osserva che la politica delle favole non si situa a monte della scrittura delle stesse, ma a valle, nelle interpretazioni delegate al lettore. La lettura politica è sempre possibile, ma è demandata alla responsabilità del destinatario. Essa si situa comunque all'interno della problematica di origine antica della parrhesia: le favole ne sono un modello in quanto si presentano come discorso di verità rivolto al principe: esso non adotta, però, la brutale franchezza dei filosofi antichi, ma il modello dell'inganno salutare, dell'equivoco e del non detto, che delegano al principe stesso la formulazione della verità su se stesso. Nelle Fables, insomma, la politica è innanzitutto un'etica del discorso.

Altri articoli affrontano questioni a cavallo tra la filosofia e la retorica. Dinah RIBARD si occupa del rapporto tra scrittura e filosofia nelle Fables, mentre Laurence GIAVARINI, prendendo spunto dall'articolo classico di Leo Spitzer sull'arte della transizione, analizza lo statuto dell'exemplum nella prima raccolta. Molto suggestivo è l'articolo in cui Boris DoNNÉ illustra una nuova ipotesi, da lui già esposta in altra sede, sulla genesi della prima raccolta di favole, che ripropone su nuove basi e in modo molto più convincente l'interpretazione politica un po' datata di René Jasinski. La Fontaine avrebbe scoperto poeticamente il genere favola in occasione del processo Fouquet. L'apologo si prestava a far passare in maniera efficace e criptica messaggi politici, Fedro aveva dato l'esempio e La Fontaine ne segue le orme in uno dei suoi testi più antichi, Le Renard et l'Écureuil. Ma l'A. estende l'interpretazione a chiave anche ai testi raccolti nelle carte di Conrart, che costituiscono un primo nucleo generativo della futura raccolta e sono quasi tutti imitati da Fedro. A questa utilizzazione politica contingente si sarebbe presto sostituita un'ipotesi di traduzione-imitazione sistematica dell'opera del favolista latino, secondo il modello delle "belles infidèles", che lascia poi spazio ad un "vagabondaggio" molto meno sistematico tra le varie raccolte esopiche di cui La Fontaine progressivamente veniva a conoscenza. Se il progetto di una "traduzione" di Fedro viene alla fine abbandonato, non si può ignorare che la strutturazione in libri delle favole con prologhi di carattere metaletterario e la stessa tonalità discorsiva che caratterizza le Fables sono un lascito del favolista latino.

6 Al contenuto sapienziale delle favole sono dedicati i due articoli di Emmanuel BURY e Charles-Olivier STIKER-MÉTRAL. Emmanuel Bury cerca di individuare i punti di tangenza tra la scrittura favolistica di La Fontaine e la tradizione dei moralisti. La scrittura morale privilegiava a inizio secolo la forma poetica e alcune moralités di La Fontaine testimoniano della persistenza di queste forme. Ma soprattutto l'elemento comune tra i moralisti e l'autore di favole è il fatto che entrambi si situano alla frontiera tra il generale - ambito della filosofia secondo Aristotele - e il particolare - ambito della storia. La favola punta a singolarizzare la verità generale che esprime. Ma nella favola 
la filosofia è soprattutto una scelta di vita, come già in Montaigne e in Charron. Da questo punto di vista è significativa la presenza di Socrate sulla soglia della raccolta: immagine del filosofo che ha portato la filosofia dal cielo alla terra. Charles-olivier Stiker-Métral rilegge invece la raccolta come illustrazione della virtù aristotelica della prudenza, basata sulla valutazione concreta di situazioni sempre diverse, che in ambito animale ha come scopo la sopravvivenza. Il modello teatrale all'opera nelle Fables è decisivo da questo punto di vista: mettendo in scena situazioni conflittuali e rappresentando icasticamente i rapporti di forza tra personaggi, crea situazioni ideali per la messa in scena della prudenza. Il lettore è invitato a sua volta a sperimentare la propria prudenza nell'interpretare la favola in modo corretto.

7 L'ermeneutica delle Fables è in gioco nei due articoli di Sabine GRUFFAT e di Céline BONHERT. Gruffat si interroga sullo statuto del simbolo nelle Fables: se la lettura simbolica del mondo è ancora viva nel XVII secolo, cessa però di essere credibile per il pubblico colto. La Fontaine si avvale dell'immagine del microcosmo, che non ha ancora perso tutto il suo senso per lui, ma si diverte a mostrare lo scarto che c'è tra l'immagine e la sua decifrazione. Insomma nelle Fables non c'è più quella trasparenza tra simbolo $\mathrm{e}$ realtà che la tradizione cinquecentesca postulava. Céline Bonhert studia invece il repertorio mitologico nelle Fables. Se fin dalla tarda Antichità esso era stato assimilato al messaggio cristiano attraverso la lettura allegorica, nell'ambito del classicismo seicentesco, la mitologia da modello della parola ispirata diventa una topica, un repertorio di figure. Che statuto hanno gli dèi nella prima raccolta di favole? Il filtro del burlesco, per quanto attenuato, finisce per livellare gli dèi equiparandoli agli altri personaggi e trasformandoli in tipi comici. La Fontaine attinge ben poco ai mitografi e alle loro letture allegoriche: i personaggi mitologici delle favole vengono tutti dalla tradizione favolistica precedente. La mitologia è presente soprattutto come componente della cultura antica e le Fables sono immerse in un universo antico rispetto al quale i riferimenti moderni fungono da anacronismo ludico. Altri articoli sono dedicati ai personaggi delle Fables: Olivier LEPLATRE si interroga sul bestiario e sul modo in cui gli animali si fanno portatori di significato nell'universo lafontainiano, analizzando nello specifico il caso della lepre, Isabelle TRIVISANI-MOREAU si occupa invece dei vegetali nella prima raccolta e Sylvie REQUEMORA-GROS fa il punto su un tema assai frequente nelle Fables, cioè il viaggio, mostrando come alla condanna ben nota del viaggio come dispersione le favole alternino una visione più positiva dello spostamento come approfondimento della conoscenza di sé, purché si tratti di un viaggio "aux rives prochaines".

8 Chiude il volume la riedizione di un interessante commento letterario e grammaticale alla raccolta del 1668 da parte di Charles NODIER. Questo testo, datato 1818, conferma la ricchezza della tradizione delle annotazioni stilistico-grammaticali sulle favole e mostra la continuità tra un autore come Nodier e interpreti settecenteschi come La Harpe e Chamfort. 